stool, the material collected is readily analysable, in principle lending itself to point-of-care tests for a wide range of indications, including infectious and inflammatory diseases of the GI tract in addition to malignancy. The device can be used as a robust means of collecting material for later analysis by a wide range of technologies.

Disclosure of Interest None Declared.

\section{PWE-009 MEASUREMENT OF COLONIC POLYPS. IS VISUAL ESTIMATION ACCURATE?}

J Woo*, H Rozati, K Besherdas. Gastroenterology, Barnet and Chase Farm Hospitals NHS Trust, London, UK

\subsection{6/gutjnl-2014-307263.269}

Introduction Colon polyp size is a critical biomarker for clinical management of colonic polyps. Larger polyps have a greater malignant potential. During colonoscopy, it is important to correctly measure the size of the polyps because of the direct correlation of size with colon cancer. ${ }^{1}$ During polypectomy, size of the colonic polyps encountered are often gauged by visual estimation or the open forceps method. ${ }^{2}$ However, some data exists on the questionable reliability of a visual estimate even amongst expert colonoscopists. We aim to compare the estimation of polyp size using the visual estimation of colon polyp with or without the open biopsy forceps technique against actual polyp size measurement by our histopathology department for all polyps $>1 \mathrm{~cm}$ in size.

Methods A single centre, retrospective analysis using the Unisoft GI auditors software was used to identify patients who have had polypectomies done for polyps $>1 \mathrm{~cm}$ in size from October 2005 till September 2013. The size of the polyps documented in the endoscopy report was then compared to the lab measured actual polyp size.

Results A total of 39 patients were identified with polyps $>1 \mathrm{~cm}$ in size who has had polypectomy done. Results are as below:

Conclusion From this study we can conclude that visual estimation with or without the open biopsy forceps technique is completely inaccurate with wide variations between the reported size and the actual size of the polyps when measured in our laboratory. Accurate measurement of colonic polyps is important as inaccuracies can lead to potentially larger polyps not being tattooed and subsequent difficulty in identification during surgery and surveillance. We advocate that the 'gold standard' practice of direct measurement of the polyp once excised and outside the body be adopted and the actual size should be documented according to direct measurement.

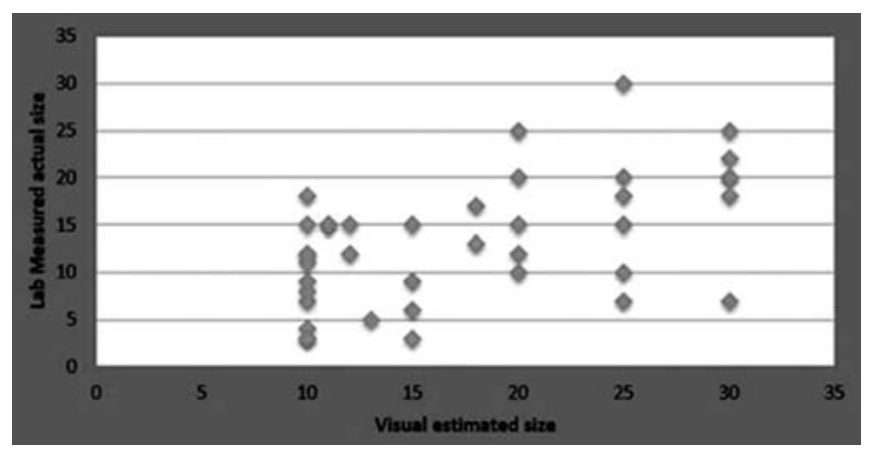

Abstract PWE-009 Figure 1

\section{REFERENCES}

1 Gopalswamy N, Shenoy V, Choudhry U et al. Is in vivo measurement of size of polyps during colonoscopy accurate? Gastrointest Endosc. 1997 Dec;46(6):497502

2 Rex D, Rabinovitz R. Variable interpretation of polyp size by using open forceps by experienced colonoscopists. Gastrointest Endosc. 2013 Oct 8. pii: S0016-5107 (13)02317-1

Disclosure of Interest None Declared.

\section{PWE-010 THE ASSOCIATION OF TGFB SIGNALLING PATHWAY GENE POLYMORPHISMS WITH COLORECTAL CANCER RISK: A META-ANALYSIS}

${ }^{1} \mathrm{JL}$ McGuire*, ${ }^{1,2} \mathrm{M}$ McPhail, ${ }^{1,2} \mathrm{~A}$ Rajendran, ${ }^{1,2} \mathrm{KJ}$ Monahan. ${ }^{1}$ Imperial College London, West Middlesex University Hospital, London, UK; ${ }^{2}$ Family History of Bowel Cancer Clinic, West Middlesex University Hospital, London, UK

\subsection{6/gutjnl-2014-307263.270}

Introduction Background

Approximately $35 \%$ of colorectal cancer risk is due to heritable factors. To date, a large fraction of this heritability remains unexplained. The TGFß signalling pathway has an increasingly implicated role in colorectal carcinogenesis, with highly penetrant-germline mutations of BMPR1A, SMAD4 and GREM1 causing known polyposis syndromes. We propose that common, low penetrance variation of TGFß signalling genes may account for much of the unexplained heritability of colorectal cancer, underlining the importance of this signalling pathway in the aetiology of colorectal cancer.

Aim A meta-analysis of the association of TGFß signalling pathway gene single nucleotide polymorphisms (SNP) with low penetrance colorectal cancer risk.

Methods A systematic literature search of Medline and Embase was performed. Data was extracted from eligible studies, according to pre-specified criteria. RevMan software, version 5.2, was used to generate pooled odds ratios (OR) to estimate the risk attributed to each variant. In addition to this, subgroup analyses for ethnicity, gender and tumour site were performed to investigate these as sources of heterogeneity.

Results Between 9,854 and 27,641 cases were meta-analysed for each SNP. Of the 10 SNPs discovered in a review of the literature, 8 were significantly associated with an increased risk of colorectal cancer in this study. These SNPs were located within BMP4, GREM1, CDH1, SMAD7, RHPN2 and BMP2, the largest effect was for rs10411210 within RHPN2 (OR=1.15; 95\% CI 1.09- 1.22, $\mathrm{I}^{2} 50 \%$ ). Subgroup analyses revealed gender as a possible source of heterogeneity, but no preferential associations for any of the SNPs with tumour site or ethnicity were detected. However determination of inconsistency between studies, i.e. $\mathrm{I}^{2}$ of $<50 \%$ for 8 of 10 SNPs, indicated that overall study heterogeneity was not a common source of bias.

Conclusion Discussion: Whilst 8 out of 10 variants showed significant association, the estimates of risk were small with all OR $<1.15$. This may result from suboptimal methods of estimating risk, as well as unknown disease heterogeneity. This process is constrained by a lack of knowledge of the true risk alleles tagged by the SNPs studied.

Conclusions The results of this analysis underline the integral role of the TGFß signalling pathway in colorectal carcinogenesis. Knowledge of the function of tagged risk alleles is required to elucidate and accurately estimate the risk attributed to polymorphisms in this pathway.

Keywords: colorectal cancer, TGF $\beta$ signalling, low penetrance Disclosure of Interest None Declared. 